\title{
Variáveis microbiológicas e produtividade do feijoeiro sob diferentes manejos do solo e calagem
}

\author{
Martha Regina Lucizano Garcia(1), Luiz Malcolm Mano de Mello(1) e Ana Maria Rodrigues Cassiolato(1)
}

(1)Universidade Estadual Paulista, Dep. de Fitossanidade, Engenharia Rural e Solos, Av. Brasil, 56, Caixa Postal 31, CEP 15385-000 Ilha Solteira, SP. E-mail: malcolm@agr.feis.unesp.br, anamaria@bio.feis.unesp.br

Resumo - O objetivo deste trabalho foi quantificar variáveis microbiológicas e produtividade do feijoeiro em razão do manejo do solo e da calagem. Parte de uma área cultivada há 20 anos em plantio direto (PD) foi submetida ao cultivo mínimo (CM), com escarificação a $0,25 \mathrm{~m}$ de profundidade, para incorporação do calcário. Os tratamentos consistiram de manejo do solo $(\mathrm{PD}$ e $\mathrm{CM})$ e 0 e $2 \mathrm{t} \mathrm{ha}^{-1}$ de calcário dolomítico aplicadas na superfície, com quatro repetições. Os maiores valores de $\mathrm{C}$ da biomassa microbiana foram verificados no $\mathrm{CM}$ e os de $\mathrm{C}$ de $\mathrm{CO}_{2}$ liberado no PD sem calagem. A menor colonização micorrízica e a maior esporulação foram observadas no $\mathrm{CM}$ sem calagem. Não foram detectadas diferenças entre os tratamentos em relação à produção de matéria seca, enquanto na produtividade de grãos, o maior valor foi verificado no CM com calagem. É possível evitar a interrupção do PD com aplicação de calcário na superfície, visto que a incorporação do material de cobertura e a calagem pouco alteraram a estabilidade do sistema, conforme comprovado pelos valores estatísticos semelhantes de $\mathrm{C}$ do $\mathrm{CO}_{2}$ liberado e pelas pequenas diferenças observadas nas demais variáveis entre manejos.

Termos para indexação: plantio direto, cultivo mínimo, carbono de biomassa microbiana, $\mathrm{CO}_{2}$ liberado, fungos micorrízicos arbusculares, cerrado.

\section{Microbial variables and productivity of bean under different soil managements and limestone application}

\begin{abstract}
The objective of this study was to quantify the microbial variables and the productivity of bean under different soil managements and limestone application. Part of a site under no-tillage (NT) system for 20 years was submitted to minimum tillage (MT), with limestone incorporation at $0.25 \mathrm{~m}$ depth and chisel plowing. The treatments were soil managements (NT and MT) and surface limestone application $\left(0\right.$ and $\left.2 \mathrm{t} \mathrm{ha}^{-1}\right)$, with four replicates. Carbon of microbial biomass higher values were found on the $\mathrm{MT}$ and $\mathrm{CO}_{2}$ evoluted, on the NT without limestone application. The smallest mycorrhizal colonization and the highest number of spores were observed on the MT without limestone application. No significant differences among treatments were detected for dry matter production. For grain yield, the higher values were found on the MT with limestone application. It is possible to avoid interruptions on the NT by the surface application of limestone, once the incorporation of organic material and limestone induced little changes on the system stability, which was confirmed by the similarity among statistical values for the $\mathrm{C}-\mathrm{CO}_{2}$ evoluted and the slight differences for the other variables studied between soil managements.
\end{abstract}

Index term: tillage system, minimum-tillage system, carbon of microbial biomass, $\mathrm{CO}_{2}$ evoluted, arbuscular mycorrhizal fungi, cerrado.

\section{Introdução}

A proteção da superfície do solo, com resíduos vegetais, é um dos meios mais efetivos para reduzir as perdas por erosão decorrentes da diminuição do impacto direto das gotas de chuva sobre o solo, da redução no selamento dos poros, da velocidade de escorrimento da enxurrada e do aumento na infiltração da água (Mannering \& Meyer, 1963).
O plantio direto visa reduzir substancialmente o processo de degradação em curso, auxiliando na recuperação e manutenção da sustentabilidade do agrossistema (Silva \& Resck, 1997). Esse manejo, por evitar o revolvimento do solo, também proporciona conservação da umidade, melhoria na estrutura, temperatura com variações mais estáveis e de menor amplitude, manutenção da reserva de nutrientes, entre outros, beneficiando não apenas o solo, mas as plantas. Apesar dos 
benefícios sobre o agrossistema, esse tipo de plantio requer cuidados por causa das alterações que algumas práticas, como a calagem, podem promover no solo. Esta prática é essencial, independentemente do sistema de cultivo adotado, para a correção da acidez do solo, especialmente os de regiões de clima tropical nos quais predominam baixos valores de $\mathrm{pH}$, bases trocáveis, $\mathrm{P} \mathrm{e}$ teores elevados de H+Al (Sá, 1997).

A quantidade de material orgânico que permanece no solo sob plantio direto é a mesma que no convencional, porém no primeiro esse material permanece na superfície e no segundo é incorporada. A incorporação favorece a aeração que, concomitantemente à introdução dos resíduos vegetais, acelera a atividade microbiana e a decomposição. A permanência da matéria orgânica na superfície, no plantio direto, reduz o contato com os microrganismos, e com isso a decomposição é mais lenta (Almeida, 1985).

Numerosos processos bioquímicos ocorrem no solo, por causa da atividade microbiana, com efeitos sobre as suas propriedades físicas e químicas e reflexos sobre o desenvolvimento das plantas, a produtividade agrícola e a qualidade do ambiente (Balota, 1997). A biomassa microbiana representa o compartimento central do ciclo de carbono no solo e, de acordo com as condições edafoclimáticas do ecossistema e da composição dos resíduos vegetais sobre sua superfície, pode funcionar como compartimento de reserva (nutrientes facilmente disponíveis) ou como catalisador na decomposição da matéria orgânica. Tanto a quantidade como a qualidade dos resíduos vegetais nos sistemas produtivos podem causar alterações na comunidade microbiana. Os microrganismos são sensíveis a essas modificações e, por isso, tornam-se adequados como indicadores biológicos. Estimativas a respeito da biomassa microbiana possibilitam associar nutrientes imobilizados com a fertilidade e potencial produtivo (Mercante, 2001).

Os microrganismos do solo são também de grande importância para a nutrição das plantas, por estabelecerem simbioses com certas espécies de plantas. As associações entre raízes e determinados fungos do solo, denominadas micorrizas, auxiliam as plantas, melhorando seu estado nutricional, proporcionando maior adequabilidade ao ecossistema, maior capacidade de sobrevivência de mudas transplantadas, redução nas perdas provocadas por fatores bióticos, maior produtividade em solos pobres e locais adversos e maior tolerância da planta aos estresses abióticos (Siqueira \& Franco, 1988). Como são fungos simbiontes obrigatórios, sua distribuição em solos cultivados é fortemente influenci- ada pela vegetação. Por meio da rotação de culturas, plantas eficientes na multiplicação dos fungos micorrízicos arbusculares (FMA) poderiam aumentar a quantidade do inóculo, favorecendo a colonização de culturas subseqüentes (Hayman, 1987).

O objetivo deste trabalho foi quantificar variáveis microbiológicas e a produtividade do feijoeiro em razão do manejo do solo e da calagem.

\section{Material e Métodos}

O experimento foi conduzido no ano de 2002 (cultivo de inverno), na Fazenda de Ensino e Pesquisa da Faculdade de Engenharia, Unesp, Campus de Ilha Solteira, no Município de Selvíria, MS. A temperatura média anual é de $24,4^{\circ} \mathrm{C}$ e a média anual pluviométrica é de $1.232,2 \mathrm{~mm}$. A classificação do solo da área foi atualizada para Latossolo Vermelho distrófico (Embrapa, 1999).

Originalmente, a área apresentava como cobertura vegetal o cerrado sensu stricto, mas atualmente está ocupada por culturas agrícolas, irrigadas por pivô central. Desde 1982 o plantio direto (PD) foi adotado como manejo e as culturas utilizadas, nos últimos três anos, foram milho ou soja no verão, e feijão no inverno. Em 2001, anteriormente à implantação da soja, a área foi cultivada com milho e, em agosto de 2002, foram aplicadas $2 \mathrm{t} \mathrm{ha}^{-1}$ de calcário dolomítico (85\% de PRNT) a lanço na superfície das parcelas correspondentes a esse tratamento. Em 22 de novembro, as parcelas foram subdivididas; uma parte foi mantida sem mobilização do solo e, a outra, submetida ao cultivo mínimo (CM), com escarificação até $0,25 \mathrm{~m}$ de profundidade e, em seguida, realizada a semeadura da soja.

O feijão foi semeado em PD, no inverno, em sucessão à soja, em 12 de maio de 2002. Na semeadura, utilizaramse $20 \mathrm{~kg} \mathrm{ha}^{-1}$ de $\mathrm{N}, 70 \mathrm{~kg} \mathrm{ha}^{-1}$ de $\mathrm{P}_{2} \mathrm{O}_{5}$ e $40 \mathrm{~kg} \mathrm{ha}^{-1}$ de $\mathrm{K}_{2} \mathrm{O}$, mediante aplicação de $250 \mathrm{~kg} \mathrm{ha}^{-1}$ da fórmula 8-28-16 e, em cobertura, 25 dias após a semeadura, aplicaram-se $200 \mathrm{~kg} \mathrm{ha}^{-1}$ da fórmula 20-0-20. A cultivar de feijão utilizada foi a IAC - Carioca, em espaçamento entrelinhas de $0,50 \mathrm{~m}$, com estande de 10 plantas por metro. O delineamento experimental foi em blocos ao acaso com parcelas subdivididas, cujos tratamentos principais foram os tipos de manejo do solo (PD e CM) e os secundários foram constituídos pelas doses de calcário (0 e $\left.2 \mathrm{t} \mathrm{ha}^{-1}\right)$ aplicadas na superfície, em um total de quatro tratamentos, com quatro repetições. Cada parcela foi composta por 14 linhas de $40 \mathrm{~m}$.

A amostragem do solo foi feita no mês de agosto de 2002, durante a fase de enchimento de grãos. Foram coletadas 20 subamostras $(0-0,10 \mathrm{~m})$ para formarem 
uma amostra composta por parcela. Depois de secadas e peneiradas, suas características químicas foram determinadas conforme Raij \& Quaggio (1983).

Nas demais determinações, cada amostra de solo foi composta de quatro subamostras por parcela, e foram coletadas na profundidade de $0-0,10 \mathrm{~m}$, na linha, próximo à raiz da planta, com auxílio de enxada. Após perda do excesso de umidade, as amostras foram peneiradas (malha de $2 \mathrm{~mm}$ ) e armazenadas. Na análise das raízes, foram coletadas plantas inteiras, e as raízes foram lavadas com água corrente e preservadas em álcool 50\%, até que a coloração fosse realizada.

Nas análises da produção da matéria seca da parte aérea do feijoeiro (com exceção dos grãos) foram retirados aproximadamente $200 \mathrm{~g}$ de plantas, de cada parcela, as quais foram colocadas em sacos de papel e tiveram imediatamente anotada sua massa úmida para, posteriormente, serem secadas em estufa a $105^{\circ} \mathrm{C}$ por 24 horas, e determinada a massa da matéria seca (MMS). As plantas colhidas na área útil de cada parcela foram pesadas para ser posteriormente calculada a produtividade de matéria seca.

Nas análises de carbono da biomassa microbiana $(\mathrm{CBM})$ e do carbono do $\mathrm{CO}_{2}\left(\mathrm{C}-\mathrm{CO}_{2}\right)$ liberado foram avaliadas quatro repetições por tratamento, sendo cada repetição constituída da média aritmética dos resultados da análise de duas amostras de solo. Para número de esporos, foram avaliadas quatro repetições por tratamento, sendo cada repetição constituída de uma amostra composta por oito subamostras de solo. Os dados foram submetidos à análise de variância pelo programa SANEST e SAS, e as médias comparadas pelo teste de Tukey a $5 \%$ de probabilidade.

O CBM foi avaliado segundo Vance et al. (1987), método que envolve a eliminação da microflora do solo pelo clorofórmio. O C liberado pela morte dos micror- ganismos foi determinado por extração seguido de digestão, comparando-se as amostras de solo não-fumigadas com as fumigadas. Na quantificação do $\mathrm{C}-\mathrm{CO}_{2}$ liberado, $100 \mathrm{~g}$ de solo foram colocados em jarros de vidro, com tampa de rosca, no centro do qual foi depositado um frasco contendo $10 \mathrm{~mL}$ de $\mathrm{NaOH} 0,1 \mathrm{~mol} \mathrm{~L}^{-1}$. Os jarros foram fechados hermeticamente e mantidos em câmara climatizada a $27^{\circ} \mathrm{C}$ por 48 horas. A titulação da soda livre foi realizada com $\mathrm{HCl} 0,1 \mathrm{~mol} \mathrm{~L}^{-1}$ e permitiu calcular, por subtração, a quantidade de $\mathrm{CO}_{2}$ que combinou com o NaOH (Anderson \& Domsch, 1982).

Nas avaliações da porcentagem de colonização por fungos FMA autóctones, as raízes foram clareadas em $\mathrm{KOH} 10 \%$, acidificadas com $\mathrm{HCl} 1 \%$, coloridas com azul de tripano $0,05 \%$ e preservadas em lactoglicerol (Phillips \& Hayman, 1970). A avaliação da colonização foi feita em 40 segmentos de $1 \mathrm{~cm}$ cada, por repetição, por tratamento, sob microscópio ótico. Na contagem dos esporos, de cada amostra de solo, $100 \mathrm{~g}$ foram processados segundo uma associação dos métodos de decantação e peneiramento úmido (Gerdemann \& Nicolson, 1963) e de centrifugação e flutuação com sacarose (Jenkins, 1964), e efetuada a quantificação dos esporos em placas com anéis concêntricos, com auxílio do microscópio estereoscópico.

\section{Resultados e Discussão}

A incorporação, pela escarificação, de parte dos resíduos vegetais e a calagem pouco alteraram as características químicas do solo. Algumas variáveis mostraram que houve efeito significativo do manejo e da calagem, mas não da interação (Tabela 1). Entre manejos, foram observadas diferenças no $\mathrm{P}, \mathrm{Al}$ e saturação por bases (V\%), sendo o maior valor de $\mathrm{Pe} \mathrm{Al}$ verificado no PD e de V\%, no cultivo mínimo. Com relação à

Tabela 1. Médias e probabilidades de F das variáveis das características químicas do solo, tomados a 0-0,10 m de profundidade, nos tratamentos com plantio direto (PD) e cultivo mínimo $(\mathrm{CM})$, com e sem aplicação de calcário na superfície ${ }^{(1)}$.

\begin{tabular}{|c|c|c|c|c|c|c|c|c|c|c|c|}
\hline Causas de variação & ariação & 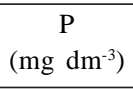 & $\begin{array}{c}\mathrm{MO} \\
\left(\mathrm{g} \mathrm{dm}^{-3}\right)\end{array}$ & $\begin{array}{c}\mathrm{pH} \\
\left(\mathrm{CaCl}_{2}\right)\end{array}$ & \multirow{2}{*}{$\begin{array}{c}\mathrm{K} \\
2,81\end{array}$} & $\mathrm{Ca}$ & $\mathrm{Mg}$ & $\mathrm{H}+\mathrm{Al}$ & $\mathrm{Al}$ & CTC & $\begin{array}{c}\mathrm{V} \\
(\%) \\
\end{array}$ \\
\hline \multirow[t]{2}{*}{ Manejo } & PD & $43,75 a$ & 30,44 & 5,21 & & 30,81 & 29,50 & 35,31 & $1,00 \mathrm{a}$ & 98,46 & $58,19 b$ \\
\hline & $\mathrm{CM}$ & $26,50 \mathrm{~b}$ & 27,13 & 5,41 & 2,19 & 32,25 & 25,37 & 30,43 & $0,50 \mathrm{~b}$ & 90,38 & $64,25 \mathrm{a}$ \\
\hline \multirow[t]{2}{*}{ Calcário } & $0 \mathrm{t} \mathrm{ha}^{-1}$ & 31,56 & 27,68 & $5,11 b$ & 2,22 & $26,94 b$ & $21,75 b$ & 34,43 & 0,87 & $85,45 b$ & $57,06 \mathrm{~b}$ \\
\hline & $2 \mathrm{t} \mathrm{ha}^{-1}$ & 38,68 & 29,87 & $5,51 \mathrm{a}$ & 2,78 & $36,13 \mathrm{a}$ & $33,12 \mathrm{a}$ & 31,31 & 0,62 & $103,39 \mathrm{a}$ & $65,37 \mathrm{a}$ \\
\hline \multicolumn{2}{|c|}{ Manejo (M) } & $0,0213^{*}$ & $0,2404^{\mathrm{ns}}$ & $0,0533^{\text {ns }}$ & $0,0807^{\mathrm{ns}}$ & $0,6910^{\mathrm{ns}}$ & $0,1573^{\text {ns }}$ & $0,0538^{\mathrm{ns}}$ & $0,0477^{*}$ & $0,1710^{\mathrm{ns}}$ & $0,0281^{*}$ \\
\hline \multicolumn{2}{|c|}{ Calcário (C) } & $0,2837^{\mathrm{ns}}$ & $0,5662^{\mathrm{ns}}$ & $0,0022^{* *}$ & $0,1111^{\mathrm{ns}}$ & $0,0261^{*}$ & $0,0025^{* *}$ & $0,1909^{\mathrm{ns}}$ & $0,2862^{\mathrm{ns}}$ & $0,0093^{* *}$ & $0,0063^{* *}$ \\
\hline \multicolumn{2}{|l|}{$\underline{M \times C}$} & $0,9522^{\mathrm{ns}}$ & $0,5443^{\mathrm{ns}}$ & $0,1931^{\mathrm{ns}}$ & $0,6640^{\mathrm{ns}}$ & $0,7640^{\mathrm{ns}}$ & $0,3140^{\mathrm{ns}}$ & $0,9089^{\mathrm{ns}}$ & $1,0000^{\mathrm{ns}}$ & $0,5439^{\mathrm{ns}}$ & $0,2808^{\mathrm{ns}}$ \\
\hline \multicolumn{2}{|l|}{$\mathrm{CV}(\%)$} & 35,59 & 18,36 & 3,52 & 25,36 & 22,13 & 19,61 & 13,50 & 58,79 & 11,57 & 7,65 \\
\hline
\end{tabular}

${ }^{(1)}$ Médias seguidas de mesma letra, na coluna e dentro de cada variável, não diferem entre si pelo teste de Tukey a $5 \%$ de probabilidade. ${ }^{\text {ns Não- }}$ significativo. * e **Significativo a $5 \%$ e a $1 \%$ de probabilidade pelo teste $\mathrm{F}$, respectivamente. 
calagem, foram verificadas diferenças de $\mathrm{pH}, \mathrm{Ca}, \mathrm{Mg}$, $\mathrm{CTC}$ e V\%, e os maiores valores foram observados no tratamento que recebeu calcário (Tabela 1). Caires et al. (2001) também verificaram que a maior dose de calcário mostrou-se mais eficiente na correção da acidez do solo.

No presente trabalho, quanto à $\mathrm{MO}$, diferenças estatísticas significativas não foram detectadas entre manejos do solo, discordando de Alvarez et al. (1995), que verificaram que esta variável foi o principal componente do solo influenciado pelo manejo convencional, e de Marchiori Júnior \& Melo (2000), que relataram alterações no teor de $\mathrm{MO}$ com o manejo do solo.

Da mesma forma, somente $\mathrm{P}$ e Al mostraram diferenças entre manejos, discordando de outros autores. Segundo Alvarez et al. (1995), embora práticas como o PD promovam maior acúmulo de nutrientes nos primeiros centímetros de solo, o menor revolvimento no PD e no $\mathrm{CM}$, em relação ao cultivo convencional, tem demonstrado que estes sistemas tendem a se igualar com o passar dos anos (Souza \& Alves, 2003). De acordo com Caires et al. (2000), esta tendência à homogeneização pode ser explicada pela não movimentação do solo, que proporciona a descida de nutrientes no seu perfil por meio de canais formados por raízes decompostas.

Neste trabalho, somente o manejo do solo interferiu nos valores de CBM, e não houve interação significativa entre manejos e calagem (Tabela 2). Diferente de resultados anteriores, foi verificado maior teor de CBM no $\mathrm{CM}$, possivelmente em razão da recente incorporação parcial dos resíduos vegetais. Segundo Vargas \&

Tabela 2. Médias e probabilidades de $\mathrm{F}$ de carbono da biomassa microbiana (CBM) e do carbono do $\mathrm{CO}_{2}\left(\mathrm{C}-\mathrm{CO}_{2}\right)$ liberado nos manejos plantio direto e cultivo mínimo, com e sem aplicação de calcário na superfície do solo ${ }^{(1)}$.

\begin{tabular}{|c|c|c|}
\hline Variáveis & 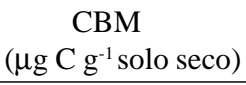 & $\begin{array}{c}\mathrm{C}-\mathrm{CO}_{2} \text { liberado } \\
\left(\mu \mathrm{g} \mathrm{CO}_{2} \mathrm{~g}^{-1} \text { solo seco } \mathrm{dia}^{-1}\right)\end{array}$ \\
\hline \multicolumn{3}{|l|}{ Manejo } \\
\hline Cultivo mínimo & $96,87 \mathrm{a}$ & 9,71 \\
\hline Plantio direto & $80,50 \mathrm{~b}$ & 10,02 \\
\hline \multicolumn{3}{|l|}{ Calcário } \\
\hline $0 \mathrm{t} \mathrm{ha}^{-1}$ & 91,62 & 10,43 \\
\hline $2 \mathrm{tha}^{-1}$ & 85,75 & 9,30 \\
\hline Manejos (M) & $0,00221^{* *}$ & $0,63159^{\text {ns }}$ \\
\hline Calcário (C) & $0,15191^{\mathrm{ns}}$ & $0,10101^{\mathrm{ns}}$ \\
\hline $\mathrm{M} \times \mathrm{C}$ & $0,63535^{\text {ns }}$ & $0,02396^{*}$ \\
\hline $\mathrm{CV}(\%)$ & 8,51 & 12,66 \\
\hline
\end{tabular}
${ }^{(1)}$ Médias seguidas da mesma letra, na coluna e dentro de cada variável,
não diferem entre si pelo teste de Tukey a $5 \%$ de probabilidade. ${ }^{\text {ns Não- }}$
significativo. $*$ e $* *$ Significativo a $5 \%$ e a $1 \%$ de probabilidade pelo teste $\mathrm{F}$, respectivamente.
Scholles (2000), no PD, com a permanência dos resíduos na superfície, sua mineralização ocorre de forma gradual, podendo promover o aumento no teor de carbono. Uma maior quantidade de C, N, P e outros nutrientes fica temporariamente imobilizada nas células microbianas, evitando perdas por lixiviação, fixação e outros (Colozzi-Filho et al., 2001). As alterações decorrentes da incorporação dos resíduos da superfície no CM foram constatadas pelos altos teores de CBM neste manejo, concordando com Alvarez et al. (1995), que afirmou que a biomasssa microbiana permite acompanhar, de forma mais rápida, as perturbações ocasionadas ao solo.

Com a escarificação, a incorporação dos resíduos promove maior aeração do solo e disponibilização de substratos, o que estimula a atividade microbiana (Salinas-Garcia et al., 1997). No entanto, neste trabalho, quanto ao teor de $\mathrm{C}-\mathrm{CO}_{2}$ liberado, não foram observadas influências do manejo ou da calagem (Tabela 2). Em relação à colonização micorrízica, os diferentes tipos de manejo e calagem interferiram no processo (Tabela 3). Na interação manejo x calagem, diferenças significativas foram verificadas entre PD e CM no tratamento que não recebeu calcário, com maior taxa observada no plantio direto (Tabela 4). Dentro de manejos, somente ocorreram diferenças no PD, com o menor teor de $\mathrm{C}-\mathrm{CO}_{2}$ liberado detectado na área que não recebeu calcário.

A quantificação do $\mathrm{C}-\mathrm{CO}_{2}$ liberado, resultante do metabolismo da maioria dos microrganismos no solo,

Tabela 3. Médias e probabilidades de F de colonização micorrízica, número de esporos de fungos micorrízicos arbusculares autóctones, matéria seca da parte aérea e produtividade de grãos, nos manejos plantio direto (PD) e cultivo mínimo (CM), com e sem aplicação de calcário na superfície do solo ${ }^{(1)}$

\begin{tabular}{|c|c|c|c|c|}
\hline Variáveis & $\begin{array}{c}\text { Colonização } \\
(\%)\end{array}$ & $\begin{array}{l}\text { № de esporos/ } \\
100 \mathrm{~g} \text { de solo }\end{array}$ & $\begin{array}{c}\text { Matéria seca } \\
\left(\mathrm{t} \mathrm{ha}^{-1}\right)\end{array}$ & $\begin{array}{c}\text { Grãos } \\
\left(\mathrm{kg} \mathrm{ha}^{-1}\right)\end{array}$ \\
\hline \multicolumn{5}{|l|}{ Manejo } \\
\hline $\mathrm{PD}$ & $100,00 \mathrm{a}$ & $26,12 b$ & 2,71 & $2.148,05 b$ \\
\hline $\mathrm{CM}$ & $99,65 b$ & $53,00 \mathrm{a}$ & 3,13 & $2.341,05 \mathrm{a}$ \\
\hline \multicolumn{5}{|l|}{ Calcário } \\
\hline $0 \mathrm{tha}^{-1}$ & $99,65 b$ & 39,75 & 2,89 & $2.209,27$ \\
\hline $2 \mathrm{t} \mathrm{ha}^{-1}$ & $100,00 \mathrm{a}$ & 39,37 & 2,95 & $2.279,82$ \\
\hline Manejo (M) & $0,03535^{*}$ & $0,00413^{* *}$ & $0,18851^{\mathrm{ns}}$ & $0,00305^{* *}$ \\
\hline Calcário (C) & $0,03535^{*}$ & $0,95724^{\mathrm{ns}}$ & $0,82871^{\mathrm{ns}}$ & $0,16770^{\mathrm{ns}}$ \\
\hline$M \times C$ & $0,03535^{*}$ & $0,66712^{\mathrm{ns}}$ & $0,27629^{\mathrm{ns}}$ & $0,01175^{*}$ \\
\hline $\mathrm{CV}(\%)$ & 0,28 & 35,25 & 19,96 & 4,21 \\
\hline
\end{tabular}

(1)Médias seguidas de mesma letra, na coluna e dentro de cada variável, não diferem entre si pelo teste de Tukey a $5 \%$ de probabilidade. ${ }^{\text {ns Não- }}$ significativo. * e **Significativo a $5 \%$ e a $1 \%$ de probabilidade pelo teste $\mathrm{F}$, respectivamente. 
tem-se mostrado uma determinação sensível (Colozzi Filho et al., 2001). O monitoramento das comunidades microbianas, por meio desta variável, tem sido utilizado como indicador da qualidade do solo em razão dos diferentes sistemas de manejo e rotações de culturas (Mercante, 2001). Ao contrário dos resultados deste trabalho, Vargas \& Scholles (2000) e Alvarez et al. (1995) verificaram que a respiração microbiana decrescia com o revolvimento, sendo influenciada pelo manejo do solo.

$\mathrm{Na}$ interação manejo x calagem, foram verificadas diferenças significativas e o menor valor foi constatado no CM sem calagem (Tabela 4). Entre manejos, foram verificadas diferenças no tratamento sem calcário, em que a maior porcentagem de colonização foi observada no plantio direto. Com relação ao número de esporos, foram detectadas diferenças significativas entre os manejos, mas não entre os níveis de calcário ou para a interação manejo x calagem (Tabela 3). O número de esporos do $\mathrm{CM}$ foi o dobro do plantio direto. A esporulação mostrou correlação positiva e significativa para $\mathrm{CBM}(0,50)$ e $\mathrm{V}(0,50)$ e correlação negativa para $\mathrm{P}(-0,67)$ e $\mathrm{H}+\mathrm{Al}(-0,51)$. Também foi verificada correlação positiva e significativa entre MMS e PROD $(0,77)$, e entre PROD e CBM $(0,50)$.

Apesar da elevada colonização observada no dois manejos, a maior esporulação em CM reforça a hipótese da maior estabilidade do sistema PD, concordando com Colozzi-Filho et al. (2001) que afirmam que a maior estabilidade do sistema micorrízico em solo sob PD devese ao não revolvimento do solo, possibilitando a permanência da rede de hifas externas e das raízes colonizadas no solo e, conseqüentemente, garantindo a

Tabela 4. Taxa de carbono do $\mathrm{CO}_{2}\left(\mathrm{C}-\mathrm{CO}_{2}\right)$ liberado, porcentagem de colonização micorrízica e produção de grãos da cultura do feijão para os manejos plantio direto e cultivo mínimo, com e sem aplicação de calcário na superfície do solo ${ }^{(1)}$.

\begin{tabular}{ccc}
\hline Calcário $\left(\mathrm{t} \mathrm{ha}^{-1}\right)$ & Plantio direto & Cultivo mínimo \\
\hline & $\mathrm{C}-\mathrm{CO}_{2}\left(\mu \mathrm{g} \mathrm{g}^{-1}\right.$ solo seco dia $\left.^{-1}\right)$ \\
0 & $11,42 \mathrm{aA}$ & $9,43 \mathrm{aB}$ \\
2 & $8,62 \mathrm{bA}$ & $9,98 \mathrm{aA}$ \\
\hline & Colonização micorrízica $(\%)$ \\
2 & $100,00 \mathrm{aA}$ & $99,30 \mathrm{bB}$ \\
0 & $100,00 \mathrm{aA}$ & $100,00 \mathrm{aA}$ \\
\hline \multicolumn{4}{c}{ Produção de grãos $\left(\mathrm{kg} \mathrm{ha}^{-1}\right)$} \\
2 & $2.186,92 \mathrm{aA}$ & $2.231,62 \mathrm{bA}$ \\
\hline
\end{tabular}

${ }^{(1)}$ Médias seguidas da mesma letra, minúscula na coluna e maiúscula na linha, dentro de cada variável, não diferem entre si pelo teste de Tukey a $5 \%$ de probabilidade. micorrização das culturas subseqüentes pela manutenção do potencial infectivo do solo. Hungria et al. (1997) relataram que a presença do feijoeiro tende a incrementar o teor de fenólicos totais no solo, o que pode conduzir à maior esporulação dos FMA.

Não foram observadas diferenças significativas na massa da matéria seca (Tabela 3), ao passo que, na produção de grãos (PROD), houve interação entre sistemas de manejos do solo e calagem, com maiores valores observados no CM com calagem (Tabelas 3 e 4). Provavelmente, tal fato seja decorrente da incorporação de parte dos resíduos vegetais, e da descompactação da superfície, aliados à calagem, que promoveram aumento na disponibilidade de nutrientes, corroborando Caires et al. (2001).

\section{Conclusões}

1. A incorporação do material de cobertura e do calcário, por meio de uma escarificação, altera pouco a estabilidade do sistema.

2. A interrupção do PD pode ser evitada pela aplicação de calcário na superfície do solo.

3. A produção de grãos é influenciada pelo manejo e interação entre manejos do solo e calagem, com maior valor no tratamento de escarificação com calagem.

\section{Referências}

ALMEIDA, F.S. de. Influências da cobertura morta do plantio direto na biologia do solo. In: FANCELLI, A.L. (Ed.). Atualização em plantio direto. Piracicaba: Fundação Cargill, 1985. p.103-144.

ALVAREZ, R.; DOAZ, R.A.; BARBERI, N.; SANTANATOGLIA, O.J.; BALOTA, L. Soil organic carbon, microbial biomass and $\mathrm{CO}_{2}-\mathrm{C}$ production from tree tillage systems. Soil \& Tillage Research, v.33, p.17-28, 1995.

ANDERSON, T.H.; DOMSCH, K.H. Ratios of microbial biomass carbon to total organic carbon in arable soils. Soil Biology \& Biochemistry, v.21, p.471-479, 1982.

BALOTA, E.L. Alterações microbiológicas em solos cultivados sob plantio direto. In: PEIXOTO, R.T. dos G.; AHRENS, D.C.; SAMAHA, M.J. (Ed.). Plantio direto: o caminho para uma agricultura sustentável. Ponta Grossa: Iapar; PRP/PG, 1997. p.5365 .

CAIRES, E.F.; BANZATO, D.A.; FONSECA, A.F. Calagem na superfície em sistema plantio direto. Revista Brasileira de Ciência do Solo, v.24, p.161-169, 2000. 
CAIRES, E.F.; FONSECA, A.F.; FELDHAUS, I.C.; BLUM, J. Crescimento radicular e nutrição da soja cultivada no sistema plantio direto em resposta ao calcário e gesso na superfície. Revista Brasileira de Ciência do Solo, v.25, p.1029-1040, 2001.

COLOZZI FILHO, A.; ANDRADE, D.S.; BALOTA, E.L. Atividade microbiana em solos cultivados em sistema plantio direto. Informe Agropecuário, v.22, p.84-91, 2001.

EMBRAPA. Centro Nacional de Pesquisa de Solos (Rio de Janeiro, RJ). Sistema brasileiro de classificação de solos. Brasília-SPI; Embrapa-CNPS, 1999. 412p.

GERDEMANN, J.W.; NICOLSON, T.H. Spores of mycorrhizal endogone species extracted from soil by wet sieving and decanting. Transactions of the British Mycological Society, v.46, p.234244, 1963.

HAYMAN, D.S. VA Micorrhyzas in field crop systems. In: SAFIR, G.R. (Ed.). Ecophysiology of VA mycorrhizal plants. Boca Raton: CRC Press, 1987. p.171-192.

HUNGRIA, M.; ANDRADE, D. de S.; COLOZZI-FILHO, A.; BALOTA, E.L. Interação entre microrganismos do solo, feijoeiro e milho em monocultura ou consórcio. Pesquisa Agropecuária Brasileira, v.32, p.807-818, 1997.

JENKINS, W.R. A rapid centrifugal-flotation technique for separating nematodes from soil. Plant Disease Reporter, v.48, p.692, 1964.

MANNERING, J.V.; MEYER, L.D. The effects of various rates of surface mulch on infiltration and erosion. Soil Science Society of America Proceedings, v.27, p.84-86, 1963.

MARCHIORI JÚNIOR, M.; MELO, W.J. de. Alterações na matéria orgânica e na biomassa microbiana em solo de mata natural submetido a diferentes manejos. Pesquisa Agropecuária Brasileira, v.35, p.1177-1182, 2000.

MERCANTE, F.M. Biomassa e atividade microbiana: indicadores da qualidade do solo. Direto no Cerrado, p.9-10, 2001.
PHILLIPS, J.M.; HAYMAN, D.S. Improved procedures for clearing roots and staining parasitic and vesicular-arbuscular mycorrhizal fungi for rapid assessment of infection. Transactions of the British Mycological Society, v.55, p.158-161, 1970.

RAIJ, B. van; QUAGGIO, J.A. Métodos de análises de solos para fins de fertilidade. Campinas: Instituto Agronômico, 1983. 31p. (Boletim Técnico, 81).

SÁ, J.C.M. Plantio direto em campos nativos. In: PEIXOTO, R.T. dos G.; AHRENS, D.C.; SAMAHA, M.J. (Ed.). Plantio direto: o caminho para uma agricultura sustentável. Ponta Grossa: Iapar; PRP/PG, 1997. p.53-65.

SALINAS-GARCIA, J.R.; HONS, F.M.; MATOCHA, J.E. Longterm effects of tillage and fertilization on soil organic matter dynamics. Soil Science Society of America Journal, v.61, p.152-159, 1997.

SILVA, J.E.; RESCK, D.V.S. Plantio direto no cerrado. In: PEIXOTO, R.T. dos G.; AHRENS, D.C.; SAMAHA, M.J. (Ed.). Plantio direto: o caminho para uma agricultura sustentável. Ponta Grossa: Iapar; PRP/PG, 1997. p.158-184.

SIQUEIRA, J.D.; FRANCO, A.A. Biotecnologia do solo: fundamentos e perspectivas. Brasília: Nagy Ltda., 1988. 235p.

SOUZA, Z.M.; ALVES M.C. Propriedades químicas de um Latossolo Vermelho distrófico de cerrado sob diferentes usos e manejos. Revista Brasileira de Ciência do Solo, v.27, p.133-139, 2003.

VANCE, E.D.; BROOKES, P.C.; JENKINSON, D.S. An extraction method for measuring soil microbial biomass C. Soil Biology \& Biochemistry, v.19, p.773-777, 1987.

VARGAS, L.K.; SCHOLLES, D. Biomassa microbiana e produção de $\mathrm{C}-\mathrm{CO}_{2}$ e $\mathrm{N}$ mineral de um Podzólico Vermelho-Escuro submetido a diferentes sistemas de manejo. Revista Brasileira de Ciência do Solo, v.24, p.35-42, 2000.

Recebido em 24 de abril de 2004 e aprovado em 12 de julho de 2004 